\title{
PD Control Strategy Design and Simulation of Magnetic Bearing with Single Freedom of Degree
}

\author{
Yang Guang \\ College of Electrical and Information Engineering \\ Beihua University \\ Jilin, China \\ e-mail: jilin313@163.com
}

\author{
Zhang Jian-Min \\ College of Electrical and Information Engineering \\ Beihua University \\ Jilin, China
}

\begin{abstract}
Based on the analysis of the model of the SingleDegree-of-Freedom (SDF) magnetic bearing system, the issue of design and simulation of PD control strategy in the system is investigated. First, the plant model of the AMB (Active Magnetic Bearings) with Single-Degree-of-Freedom (SDF) is found out to be unstable plant. Then, based on the root locus theory and Routh stability criteria, the necessity of derivation action in the controller is analyzed.In addition, the PD control strategy for a particular plant is designed, the effectiveness of which is validated by the given simulation examples. The proposed approach can provide an important reference for the practical application of PD control strategy in the magnetic bearing system.
\end{abstract}

Keywords- Magnetic Bearing; PD; Control Strategy; Design; Simulation

\section{INTRODUCTION}

The electromagnetic levitation technology belongs to automatic control technology and it has been built with the development of automatic control technology. The basic idea of electromagnetic levitation is to keep some item stay at a given fixed position around an axis or multiple axises. Since there is not any contact between the suspensions and the supported item, the energy consumption and speed limitation brought by friction are all moved away. Thus, due to its many advantages, including long lifespan, low energy consumption, pollution free, noiseless, none speed limitation, high safety and reliability, and so on, the electromagnetic levitation control system has become a world-wide focus at present [1].

The electromagnetic levitation system is a complex system, involved in several subjects, such as mechanical engineering, dynamics, electrical engineering, electronics, control engineering, computer science, and so on. Compared with traditional contact-type bearings, the AMB (short for Active Magnetic Bearings) have many prominent features, for instance, there is no friction loss, no friction consumption, no lubrication. Therefore, it can reach a substantially high speed in high temperature environment and its lifespan is very long, which makes it find a widely applications in practical engineering. The AMB have often been employed to replace the traditional mechanical bearings in many kinds of rotary machines, and thus the total performance of the system can be greatly improved [2]. At present, the application of many control strategies, including PID [3], LQR[4], Hळ[5], Gain-scheduling[6], fuzzy control, adaptive control, iteration learning control and robust $\mu$-synthesis [7] has been investigated.

However, since the PID control strategy remain play the dominant role in the control of $\mathrm{AMB}$, the designing issue of PD control strategy for the $A M B$ with single freedom of degree is studied from the prospective of root locus and stability criteria.

This paper is arranged as follows: Section 2 is devoted to the mathematical model development of AMB with single degree of freedom. Section 3 deals with the designing approach of PD control strategy for the AMB. Then, simulation results are given in Section 4. Finally, Section 5 concludes the paper.

\section{MODEL OF AMB WITH SINGLE DEGREE OF} FREEDOM

Consider the block diagram of AMB with single degree of freedom in [3] shown in Fig.1.

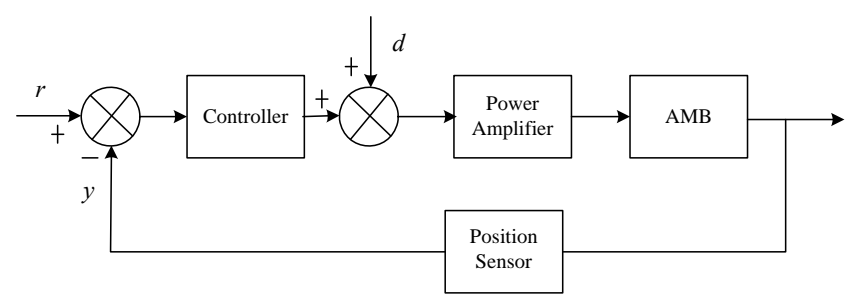

Figure 1. Block diagram of AMB control system.

The mathematical model of AMB with single degree of freedom has been obtained in [3], where the transfer function of the controlled plant - AMB is:

$$
G(s)=\frac{K_{I}}{s^{2}-K_{Z}}
$$

where $K_{I}$ is the current coefficient and $K_{Z}$ is the position coefficient. As shown in Fig.1, the power amplifier can be regarded as a proportional element with gain as $K_{a}$, and the position sensor can also be viewed as a proportional element with gain as $K_{s}$. The aim of the controller designing to form a closed-loop is to make the controlled variable position $y$ track the reference input $r$ quickly in the presence of disturbance $d$.

Consider an AMB with parameters shown in Table I:

Table I. Parameters of AMB. 


\begin{tabular}{|c|c|c|}
\hline Parameters & Sign & Value \\
\hline Rotator mass & $M$ & $1 \mathrm{~kg}$ \\
\hline Air gap & $\delta$ & $0.5 \mathrm{~mm}$ \\
\hline Power Amplifier gain & $K_{a}$ & 3.3 \\
\hline Sensor coefficient & $K_{s}$ & 8000 \\
\hline Current coefficient & $K_{I}$ & $179.86 \mathrm{~N} / \mathrm{A}$ \\
\hline Position coefficient & $K_{z}$ & $359718.4 \mathrm{~N} / \mathrm{m}$ \\
\hline
\end{tabular}

Thus, the transfer function of the AMB can be obtained based on Eq.(1) and Table I as follows:

$$
G(s)=\frac{179.86}{s^{2}-359718.4}
$$

By combination of the power amplifier and sensor element, the generalized controlled process can be obtained as:

$$
G_{p}(s)=3.3 * \frac{179.86}{s^{2}-359718.4} * 8000=\frac{4748304}{s^{2}-359718.4}=\frac{1}{2.106 \mathrm{e}-7 s^{2}-0.0758}
$$

It is obvious to find that there are two poles in the generalized controlled process, +599.7653 and -599.7653 . Since it has a role placed in the right half plane, it is an unstable plant. So we have to design an appropriate controller to form a closed-loop so as to improve the system performance.

\section{PD CONTROL STRATEGY DESIGN FOR AMB}

In practical engineering, the PID controller has a great number of advantages, including simple structure, intuitional and obvious physical meanings, strong robustness, and so on, and thus it has found a widely applications in various fields, not to mention the AMB. Based on the mathematical model developed in the last section, the designing approach of PD control strategy for the AMB system is proposed based on the root locus theory and Routh stability criteria.

Consider the generalized controlled plant shown in Eq.(3), it is easy to conclude that:

(1) If the P only controller is employed, the poles of the open loop transfer function remains +599.7653 and 599.7653, and the system response remains divergence. This response is undesirable and thus the system can become stable provided the derivation action is added.

(2) Since the pure derivation action can no be solely used, the PD controller is adopted here. The derivative action is actually ahead of time and the instantaneous error rate can be measured by the action. And then an appropriate anti-action will be produced to deal with the overlarge overshoot.

The transfer function of a PD controller is written as:

$$
G_{c}(s)=K_{p}\left(1+T_{d} s\right)=K_{p}+K_{d} s
$$

where $K_{p}$ is the proportional gain and $T_{d}$ is the derivation time constant, and $K_{d}$ is the derivation gain. And thus the open loop transfer function of the system can be obtained by combination of Eq. (3):

$$
G_{k}(s)=G_{c}(s) G_{p}(s)=\frac{K_{p}+K_{d} s}{2.106 \mathrm{e}-7 s^{2}-0.0758}=\frac{K_{d}(s-z)}{2.106 \mathrm{e}-7 s^{2}-0.0758}
$$

where $K_{p}=-z K_{d}, z$ is the zero of the open loop transfer function to be placed. Without a PD controller, the closed loop characteristic equation is written as: $2.106 \mathrm{e}-7 s^{2}-0.0758=0$, which indicates that it has two roles: one is +599.7653 , and another is -599.7653 . With a PD controller, the closed loop characteristic equation becomes: $2.106 \mathrm{e}-7 s^{2}+K_{d} s+K_{p}-0.0758=0$.Thus, in order to keep the closed-loop control system stable, a zero -1000 can be added to the open loop transfer function. For instance, if the proportional gain is chosen as $K_{p}=0.1$, then we have $K_{d}=0.0001$.

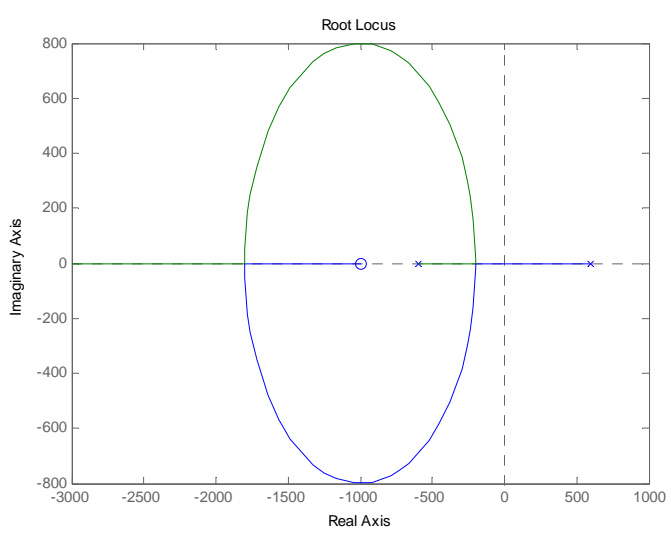

Figure 2. Root locus of system with PD controller.

As shown in Fig.2, the root locus of the closed-loop can be placed in the left half plane provided the proportional gain $K_{p}$ satisfies a certain condition. i.e., it can make all the characteristic roots have negative real part if and only if $K_{p}>0.0758$.

The closed-loop system error transfer function with respect to the reference input is:

$$
\frac{E(s)}{R(s)}=\frac{1}{1+G_{c}(s) G_{p}(s)}=\frac{2.106 \mathrm{e}-7 s^{2}-0.0758}{2.106 \mathrm{e}-7 s^{2}+K_{d} s+K_{p}-0.0758}
$$

(6)

For a unit step reference input, the steady state error is: $e_{s s}=\lim _{s \rightarrow 0} s \frac{1}{s} \frac{E(s)}{R(s)}=\lim _{s \rightarrow 0} \frac{2.106 \mathrm{e}-7 s^{2}-0.0758}{2.106 \mathrm{e}-7 s^{2}+K_{d} s+K_{p}-0.0758}=\frac{-0.0758}{K_{p}-0.0758}$ 7)

where $e_{s s}<0$, the absolute value of $e_{s s}$ can be reduced if the value of $K_{p}$ increases, and then a satisfied overshoot can be obtained.

The closed-loop error with respect to the disturbance input can be written as:

$$
\frac{E(s)}{D(s)}=\frac{-G_{p}(s)}{1+G_{c}(s) G_{p}(s)}=\frac{-\frac{1}{2.106 \mathrm{e}-7 s^{2}-0.0758}}{1+\frac{K_{p}+K_{d} s}{2.106 \mathrm{e}-7 s^{2}-0.0758}}=\frac{-1}{2.106 \mathrm{e}-7 s^{2}+K_{d} s+K_{p}-0.0758}
$$

(8)

For a unit step disturbance input, the steady state error is: 
$e_{s s}=\lim _{s \rightarrow 0} s \frac{1}{s} \frac{E(s)}{D(s)}=\lim _{s \rightarrow 0} \frac{-1}{2.106 \mathrm{e}-7 s^{2}+K_{d} s+K_{p}-0.0758}=\frac{-1}{K_{p}-0.0758}$

where $e_{s s}<0$, the absolute value of $e_{s s}$ can be reduced if the value of $K_{p}$ increases, and then a satisfied overshoot can be obtained.

\section{SIMULATION EXAMPLES}

Based on the proposed PD control strategy, three different PD parameters are chosen to obtain the unit step response as shown in Fig.3.

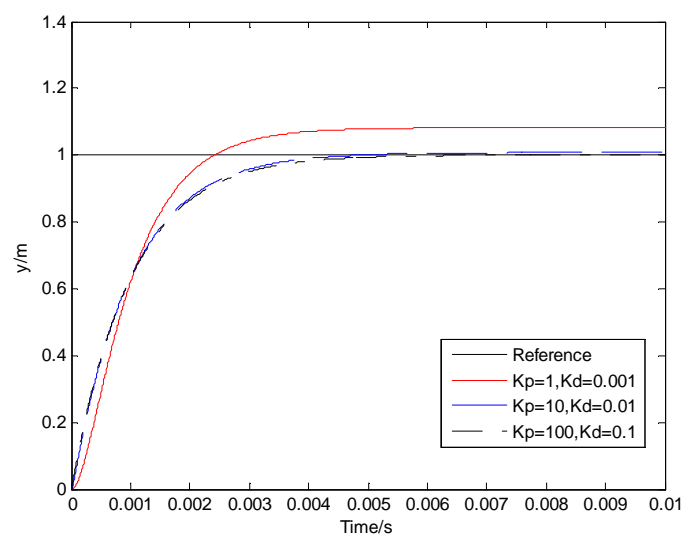

Figure 3. Unit step responses of AMB system with different PD parameters.

As presented in Fig.3, the system quickly tends to be stable with the proposed PD controllers. In addition, with the increasing of $K_{p}$, the system performance becomes better. The performance indices of the system with the three PD parameters are shown in Table 2.

Table II. Performance indices of unit step responses with different PD

\begin{tabular}{|c|c|c|c|}
\multicolumn{5}{c|}{ parameters. } \\
\hline PD parameters & $t_{r}$ & $\delta \%$ & $e_{s s}$ \\
\hline$K_{p}=1, K_{d}=0.001$ & 0.0015 & $8.196 \%$ & -0.0820 \\
\hline$K_{p}=10, K_{d}=0.01$ & 0.0021 & $0.7642 \%$ & -0.0076 \\
\hline$K_{p}=100, K_{d}=0.1$ & 0.0022 & $0.0711 \%$ & $7.5857 \mathrm{e}-4$ \\
\hline
\end{tabular}

As presented in Table II, with the increasing of $K_{p}$, the overshoot substantially reduces, the steady state error also sharply decreases. The computational results of the performance indices keep in accordance with the result obtained in Eq. (7). When $K_{p}=100$ and $K_{d}=0.1$, the steady state error of the system becomes $7.5857 \mathrm{e}-4$, nearly can be omitted, and then the design specification can be satisfied.

In addition, the unit step disturbance responses of the system with the three groups of PD controllers are shown in Fig.4.

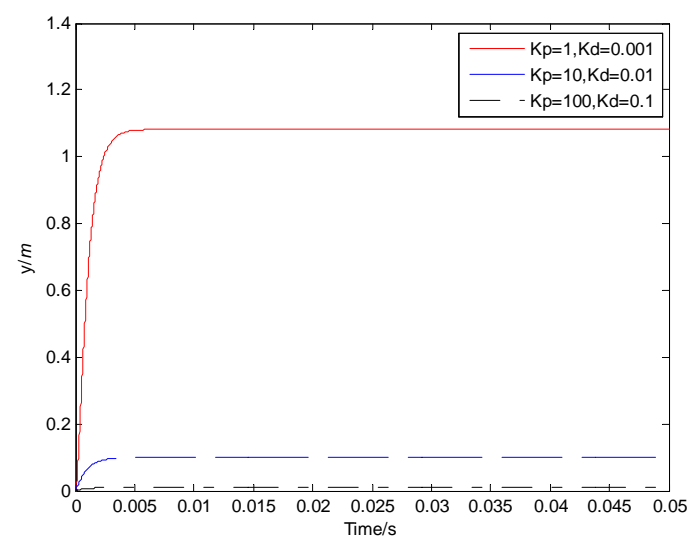

Figure 4. Unit disturbance responses of AMB system with different PD parameters.

As presented in Fig.4, the system quickly returns to be stable with the proposed PD controllers in the presence of disturbance in the control loop. Furthermore, with the increasing of $K_{p}$, the system performance become better. The performance indices of the system with the three PD parameters are shown in Table III.

Table III. Performance indices of disturbance responses with different PD parameters.

\begin{tabular}{|c|c|}
\hline PD parameters & $e_{s s}$ \\
\hline$K_{p}=1, K_{d}=0.001$ & -1.082 \\
\hline$K_{p}=10, K_{d}=0.01$ & -0.1008 \\
\hline$K_{p}=100, K_{d}=0.1$ & -0.01 \\
\hline
\end{tabular}

As presented in Table III, with the increasing of $K_{p}$, the steady state error also sharply decreases. The computational results of the performance indices keep in accordance with the result obtained in Eq. (9). In order to have a satisfied reference input tracking ability and disturbance attenuating ability, we can choose $K_{p}=100$, $K_{d}=0.1$.

\section{CONCLUSIONS}

Based on the mathematical model of AMB with single degree of freedom, the designing approach of PD control strategy is proposed based on the root locus theory and Routh stability criteria, which open a new way and method for the design and application of PID control strategy in the AMB control system. Simulation examples are given to illustrate the effectiveness of the proposed approach. However, the integral action is necessary to eliminate the steady state error, so how to add the integral action in the controller remains further investigated. In addition, how to design other advanced control strategies to deal with the AMB control system, will also one of the future research directions.

\section{REFERENCES}

[1] Yu Lie, “Controllable electromagnetic levitation system”,Beijing: Science Press, 2003.

[2] Zeng Li, Zhu Yi, Zeng Xueming, "Research on the model of hybrid magnetic bearings control system with single degree of freedom, 
Journal of Nanjing University of Aeronautics and Astronautics, 1998, vol.30, no.6, pp.685-690.

[3] Qiu Zhenxing, "Research on PID control of magnetic bearings.Master thesis”, Beijing Jiaotong University, December 2010.

[4] Guang-Ren Duan, Howe D, "Robust magnetic bearing control via eigenstructure assignment dynamical compensation”, IEEE Transactions on Control Systems Technology, 2003, pp.204-215.
[5] Ritonja, J, “Active magnetic bearing control. Ventil”, 2006, vol.12, no.1, pp.19-24.

[6] Min Sig Kang, "Sliding mode control with disturbance observer for an active magnetic bearing system”, Transactions of the Korean Institute of Electrical Engineers, 2004, vol.53, no.6, pp.408-414.

[7] Min Sig Kang Woo Hyun Yoon, "Acceleration feedforward control in active magnetic bearing system subject to base motion by filteredX LMS algorithm", IEEE Transactions on Control Systems Technology, 2006, vol14, no.1, pp.134 -140. 